\title{
Characteristic Parameters of Liquid Crystal Cells and Their Measurements
}

\author{
S. T. Tang and Hoi-Sing Kwok, Fellow, IEEE
}

\begin{abstract}
The concept of equivalent characteristic parameters of a liquid crystal (LC) cell is introduced together with the characteristic circle and the characteristic ellipse. They are all related to the twist angle and retardation of the $\mathrm{LC}$ cell. Such equivalent characteristic parameters can provide a framework for analyzing $L C$ modes, as well as providing a means to measure $L C$ cell parameters. A new Stokes parameter method is also introduced to measure these characteristic parameters. Thus the twist angle and retardation of the $\mathrm{LC}$ cell can be determined accurately.
\end{abstract}

Index Terms-Characteristic parameters, liquid crystal (LC) measurement, retardation, stokes parameter, twist angle.

\section{INTRODUCTION}

$\mathbf{I}$ T IS WELL KNOWN that lossless optical systems consist of waveplates and polarization rotators are represented by unitary matrices [1], and that for every unitary optical media, there is an optically equivalent model consisting of one waveplate followed by one polarization rotator [2]. In particular, the general twisted nematic (GTN) liquid crystal (LC) layer is a unitary system and therefore can be represented by the combination of an equivalent waveplate $W P(\Gamma, \alpha)$ and a polarization rotator $R(\chi)$, where $\alpha$ is the angle of the slow axis, $\Gamma$ is the retardation value, and $\chi$ is the rotation angle. If the Jones matrix for the LC cell is $M$, then $M=R(\chi) W P(\Gamma, \alpha)$.

There are in general three independent characteristic parameters in the equivalent model. These three parameters, $(\Gamma, \alpha, \chi)$, are functions of the incident wavelength. Therefore, a monochromatic light source has to be assumed in the discussions of the equivalence theorem in this paper.

In the design and fabrication of LC displays, the twist angle $\phi$ and LC cell retardation $d \Delta n$ are the two main design parameters. In the Jones matrix format, for simplicity, a GTN matrix usually assumes the input director to be parallel to the $x$-axis. In the following discussions, this coordinate system is assumed (Fig. 1), therefore there are only two cell parameters concerned in a GTN cell, namely $(\phi, d \Delta n)$. There have been some methods proposed for the determination of LC cell parameters, including spectral methods [3]-[5] and monochromatic light methods [6]-[9]. Recently, we have published a paper on the spectral methods [10], the methods described in that paper depend on the polarization transformation equations. In this paper only the monochromatic methods are concerned. The

Manuscript received September 22, 2005; revised November 8, 2005. This work is supported by the Hong Kong Government Innovations and Technology Fund.

The authors are with the Centre for Display Research, Department of Electrical and Electronic Engineering, Hong Kong University of Science and Technology, Clear Water Bay, Hong Kong (e-mail: eekwok@ust.hk).

Digital Object Identifier 10.1109/JDT.2005.862197 monochromatic methods have an advantage that there is no limited solution band [10]. That is for every unitary system (or LC cell), there is an equivalent optical model and that the characteristic parameters can be solved by the methods introduced in the sections followed. Consider the characteristic parameters, there has been also some methods proposed for their determination [11]-[13]. However, their relations to the determination of LC cell parameters are not well elaborated.

In Section II, the relations between the LC cell parameters $(\phi, d \Delta n)$ and the equivalent model characteristic parameters $(\Gamma, \alpha, \chi)$ are derived, and in Section III methods for determination of the characteristic parameters are introduced. In Section IV, the LC cell parameters are determined by known characteristic parameters. In Section V, a new approach to solve the twist angle and retardation of a GTN cell that bases on the Stokes parameter measurement are introduced. Two new characteristic parameters, the characteristic circle and ellipse, are discussed with measurement examples.

\section{Characteristic PARAmeters AND LC CELL PARAMETERS}

In his classical text on integrated photo-elasticity [13] and other publications [14], [15], Hillar Aben was the first to give a detail account on the theory of characteristic parameters in three-dimensional photo-elastic media. Equations for the treatment of media with uniform rotation of the principle direction were derived. However, those notations are not familiar to the LC community. In this section, we shall derive once again the relations between the equivalent model characteristic parameters $(\Gamma, \alpha, \chi)$ and the LC cell parameters $(\phi, d \Delta n)$ in a readily recognizable and applicable way. These equations are directly related to the measurement of LC cell parameters.

Suppose the Jones matrix of a unitary optical media $U_{J}$ is

$$
U_{J}=\left(\begin{array}{cc}
a-i b & -c-i d \\
c-i d & a+i b
\end{array}\right)
$$

Here $a, b, c, d$ are functions of the LC cell parameters, $\phi$ and $d \Delta n$ as

$$
\begin{aligned}
& a=\cos \beta \cos \phi+\frac{\phi}{\beta} \sin \beta \sin \phi \\
& b=\frac{\delta}{\beta} \sin \beta \cos \phi \\
& c=\cos \beta \sin \phi-\frac{\phi}{\beta} \sin \beta \cos \phi \\
& d=\frac{\delta}{\beta} \sin \beta \sin \phi
\end{aligned}
$$




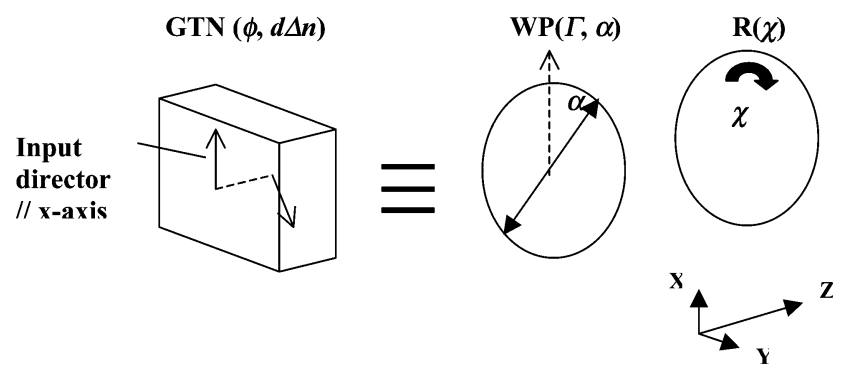

Fig. 1. A general twisted nematic cell can be treated optically equivalent to one waveplate and one polarization rotator. The waveplate azimuth $\alpha$ is relative to the LC cell input director.

where $\beta=\sqrt{\delta^{2}+\phi^{2}}, \delta=\pi d \Delta n / \lambda$ and $\phi$ is the twist angle, $\Delta n$ is the LC birefringence and $\lambda$ is the incident light wavelength [16], [17].

By the optically equivalent model (see Fig. 1), (1) is then written as

$$
U_{J}(a, b, c, d)=R(\chi) \bullet W P(\Gamma, \alpha)
$$

where $W P(\Gamma, \alpha)=R(\alpha) \bullet W P(\Gamma) \bullet R(-\alpha)$ is the Jones matrix of a waveplate with phase angle $\Gamma$ and the slow axis at $\alpha$ degree to the $x$-axis, and $R(\chi)$ is the polarization rotator with rotation angle $\chi$. The Jones matrix of $W P(\Gamma)$ and $R(\theta)$ are [18], [19]

$$
\begin{aligned}
W P(\Gamma) & =\left(\begin{array}{cc}
e^{-i \Gamma / 2} & 0 \\
0 & e^{i \Gamma / 2}
\end{array}\right) \\
R(\theta) & =\left(\begin{array}{cc}
\cos \theta & -\sin \theta \\
\sin \theta & \cos \theta
\end{array}\right) .
\end{aligned}
$$

Equating (1) and (2) gives the following relations between unitary media parameters $(a, b, c, d)$ and the characteristic parameters $(\Gamma, \alpha, \chi)$ :

$$
\begin{aligned}
& a=\cos \left(\frac{\Gamma}{2}\right) \cos \chi \\
& b=\sin \left(\frac{\Gamma}{2}\right) \cos (\chi+2 \alpha) \\
& c=\cos \left(\frac{\Gamma}{2}\right) \sin \chi \\
& d=\sin \left(\frac{\Gamma}{2}\right) \sin (\chi+2 \alpha) .
\end{aligned}
$$

and

$$
\begin{aligned}
\tan 2 \alpha & =\frac{(a d-b c)}{(a b+c d)} \\
\cos ^{2}\left(\frac{\Gamma}{2}\right) & =\left(a^{2}+c^{2}\right) \\
\tan \chi & =\frac{c}{a} .
\end{aligned}
$$

Substituting (2) into (6a)-(6c) gives

$$
\begin{aligned}
\tan 2 \alpha & =\left(\frac{\phi}{\beta}\right) \tan \beta \\
\sin ^{2}\left(\frac{\Gamma}{2}\right) & =\left(\frac{\delta^{2}}{\beta^{2}}\right) \sin ^{2} \beta \\
\tan \chi & =\frac{(\beta \sin \phi \cos \beta-\phi \cos \phi \sin \beta)}{(\beta \cos \phi \cos \beta+\phi \sin \phi \sin \beta)} .
\end{aligned}
$$

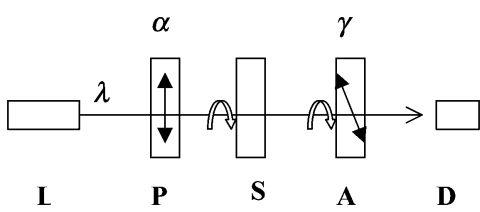

Fig. 2. Experimental setup for characteristic angle measurement. It is a common P-S-A setup. L-Monochromatic light source, P-Polarizer, S-Sample (LCD), A-Analyzer, D-Detector. $\lambda$ is the monochromatic light wavelength, $\alpha$ and $\gamma$ are the polarizer and analyzer angle respectively.

In our previous analysis [3], [10], we have pointed out that, for a GTN cell, there are two linear output solutions for a linear polarized input. The first one is the polarization rotator solution (or LP1 solution). In the equivalent model, this corresponds to a full-wave equivalent wave-plate and the rotator angle $\chi$ is equal to the twist angle $\phi$. The second linear output solution (or LP2 solution) is given by (7a). It corresponds to the linear polarized input parallel to the equivalent wave-plate azimuth $\alpha$. This input polarized light directions are named by Aben the primary characteristic directions and they are an orthogonal pair. It is important to note that one can always find a solution for (7a) and the linear output directions are named the secondary characteristic directions. The difference between the primary and secondary characteristic directions is the characteristic angle $\chi$ of the equivalent rotator.

Equations (7a)-(7c) relates the two LC cell parameters to the three equivalent model characteristic parameters. In general, the two LC cell parameters $(\phi, d \Delta n)$ are unknown and the three characteristic parameters $(\Gamma, \alpha, \chi)$ are measurables. Therefore, by knowing any two of the characteristic parameters, the two cell parameters can be solved.

\section{MEASUREMENT OF CHARACTERISTIC PARAMETERS}

There have been some methods proposed for the determination of characteristic parameters [11]-[13]. According to our experiences, the simplest method to determine the characteristic angle $\chi$ is the iterative method of Srinath and Keshavan [20]. And a modified Senarmont method is suitable for determining the characteristic phase $\Gamma$. When determining the characteristic angle $\chi$, the characteristic direction $\alpha$ can also be determined if the test cell input director direction is known. It should be noted that even though we cannot determine the angle $\alpha$, we can still determine $(\Gamma, \chi)$ and thus $(\phi, d \Delta n)$ by $(7 \mathrm{~b})$ and $(7 \mathrm{c})$. Below we shall describe briefly the experimental procedures.

\section{A. Characteristic Angle $\chi$}

The experimental setup is shown in Fig. 2. The unitary optical media and the analyzer are mounted on rotary stages. The two rotary stages were rotated iterately until a minimum transmission (should be zero theoretically) is obtained. Then the polarizer direction is the primary characteristic direction $\alpha$, the analyzer direction is the secondary characteristic direction $\pm 90^{\circ}$, the angle between the analyzer and the polarizer is the characteristic angle $\chi \pm 90^{\circ}$.

By using this iterative procedure, the final analyzer direction converges [23] by a factor approximately equal to $\sin ^{2}(\Gamma / 2)$. Usually 2 to 3 iterations will arrive at the final analyzer position. 


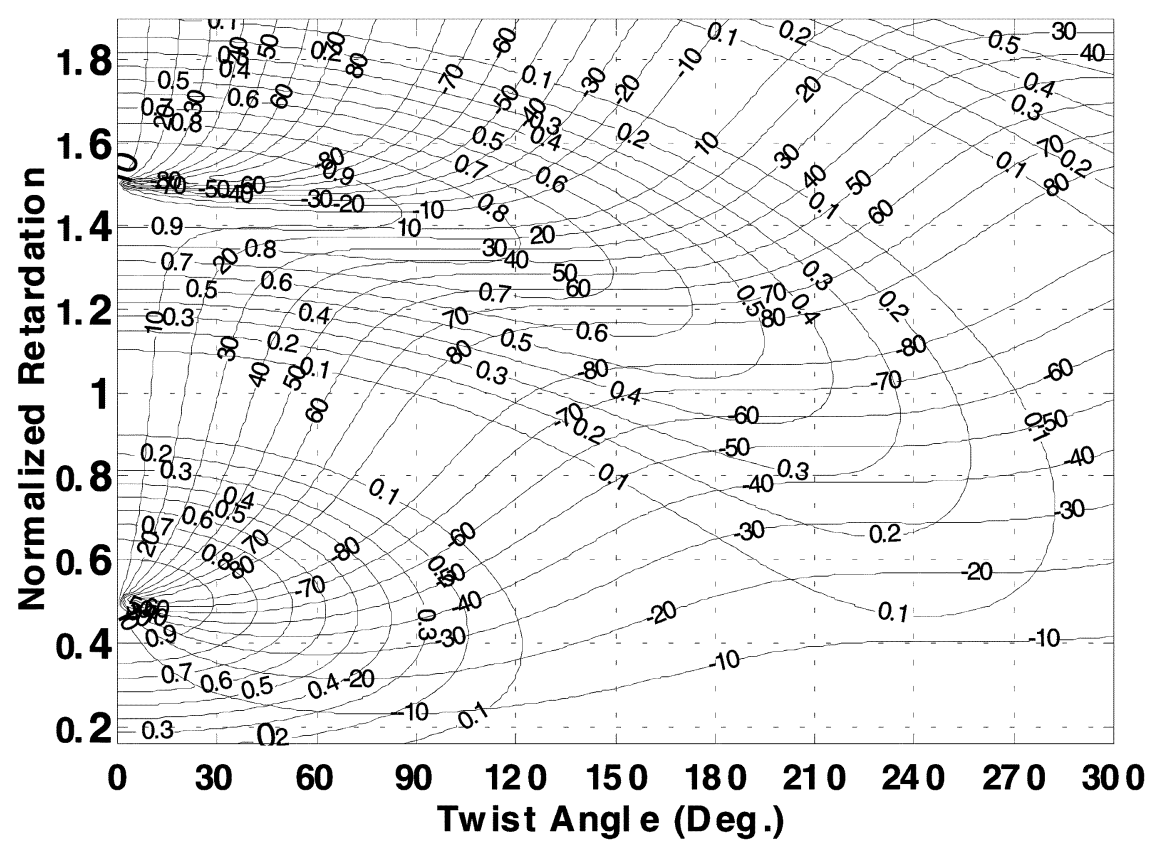

Fig. 3. A nomograph of (7b) and (7c). This graph consists of two contour plots, the first one ranges from 0 to 1 represents the right-hand side of (7b). The second one ranges from $-90^{\circ}$ to $90^{\circ}$, represents the characteristic angle $\chi$ in (7c). Normalized retardation here is $d \Delta n / \lambda$.

For a typical STN with $\Gamma=51^{\circ}$, three iterations will achieve an accuracy of 0.0064 .

\section{B. Characteristic Phase $\Gamma$}

The conventional Sernamont method of finding the phase value of a waveplate is adopted. It is a polarizer-unknown waveplate-QWP-analyzer set-up. The input polarizer is set parallel to the QWP and then the unknown waveplate is oriented $45^{\circ}$ to the input polarizer. The analyzer is then rotated to find the null or maximum transmission direction. The unknown waveplate phase angle $\Gamma$ is equal to two times the angle between the maximum transmission analyzer direction and the input polarizer direction. By using Stokes calculus, the above procedure can be expressed as

$$
S=M_{Q W P} \bullet M_{W P}\left(\Gamma, \frac{\pi}{4}\right) \bullet P_{H} .
$$

Here $P_{H}$ is the Stokes vector of a linear polarized light at horizontal. $M_{Q W P}$ and $M_{W P}$ are the Mueller matrix of a quarterwave plate and an unkown waveplate with phase $\Gamma$ and at $45^{\circ}$. The final Stokes vector before the analyzer is, therefore, $S=$ $\left(\begin{array}{lll}1 & \cos \Gamma \sin \Gamma \quad 0\end{array}\right)^{T}$. It is clear that one can get maximum transmission at an analyzer angle of $\Gamma / 2$.

To obtain the equivalent waveplate characteristic phase $\Gamma$, we employ a modified Sernamont method. The initial set-up is the same as in Fig. 2. The equivalent waveplate axis is again set $45^{\circ}$ to the input polarizer. Then the following procedure can be used to obtain the characteristic phase.

1) Complete the procedure to obtain the characteristic angle $\chi$ first.

2) Further rotate the optical media by $45^{\circ}$.

3) Insert a quarter-wave plate (QWP) in between the optical media parallel and the analyzer. The QWP axis is parallel to the analyzer. That is at $\chi$ angle to the input polarizer.
4) Rotate the analyzer to get the minimum transmission (should be zero theoretically). Rotate $\pm 90^{\circ}$ to get maximum transmission direction.

5) The angle between the QWP and the analyzer is equal to half the characteristic phase $\Gamma$.

\section{Determination of LC Cell Parameters}

In a general problem of unknown GTN cell, one way to solve the cell parameters $(\phi, d \Delta n)$ is to get the characteristic parameters $(\Gamma, \chi)$ and then solve (7b) and (7c) simultaneously. Fig. 3 is a nomograph describing the relation between $(\phi, d \Delta n / \lambda)$ and $\left(\sin ^{2} \Gamma / 2, \chi\right)$. Here the retardation is normalized by the incident wavelength to facilitate different incident wavelength. It is important to note here that the sign of the characteristic angle $\chi$ is critical. It includes information on the twist sense of the GTN cell. In Fig. 3 the twist angles are assumed to be in certain sense, characteristic angle of the same sense are said to be positive and vice versa.

Example 1: STN Cell: A super-twisted nematic (STN) LC cell was measured by the methods described in Section III. The phase angle was determined to be $\Gamma=51^{\circ}$. The angle between the polarizer and the analyzer is $85^{\circ}$. Whether it is $\pm 85^{\circ}$ depends on the STN cell twist sense. If $\chi=-85^{\circ}$ (that is, it has opposite sense to the twist angle) and $\Gamma=51^{\circ}$, it corresponds to a twist angle $\phi=-241.2^{\circ}$ and retardation $d \Delta n=$ $731 \mathrm{~nm}(\lambda=632.8 \mathrm{~nm})$. If $\chi=+85^{\circ}$, then there is another solution of $(\phi, d \Delta n)=\left(234.4^{\circ}, 771 \mathrm{~nm}\right)$ (see Figs. 3 and 4$)$. The sign of the characteristic angle cannot be determined readily if the twist sense of the GTN cell is unknown. Therefore, one more test has to be carried out. For a given GTN cell, the twist angle is fixed and if the normalized retardation varies, the solution in the nomograph will move vertically. Recall that the normalized retardation is $d \Delta n / \lambda$, the value depends on both the input light 


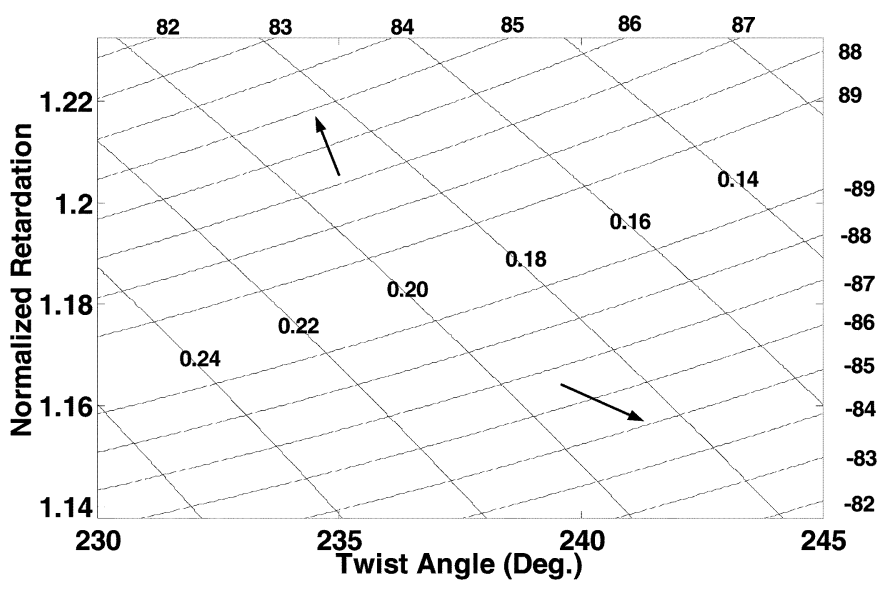

Fig. 4. Part of Fig. 3 is enlarged. The two possible solutions for $\left(\chi, \sin ^{2} \Gamma / 2\right)=\left(\left|85^{\circ}\right|, 0.185\right)$ are pointed out. It is clear that for elevated temperature, one solution will have its characteristic angle amplitude decrease and the other increase.

wavelength and temperature. Therefore, a simple method to distinguish the two possible solutions is to measure the characteristic angle again at an elevated temperature. Remember that the normalized retardation should decrease with temperature [21], therefore if $\chi$ decrease, it should have a negative sign and vice versa. By heating the LC sample with a heat blower, an experiment was carried out and the characteristic angle $\chi$ was found to decrease to $81^{\circ}$ quickly. Therefore, the original characteristic angle can be determined to be $\chi=-85^{\circ}$ and the solution was concluded to be $(\phi, d \Delta n)=\left(-241.2^{\circ}, 731 \mathrm{~nm}\right)$. In this example, it is demonstrated that not only twist angle and retardation, but also the twist sense was determined. The negative sign before the twist angle indicates a left-hand twisted cell.

In the above example, both the STN cell twist angle and retardation were assumed to be unknown. However, in the manufacturing process, the twist angle is usually well controlled, but only the cell gap (or retardation) is not confirmed. In this case, the retardation can be determined in a rather simple way.

Example 2: TN Cell With Known Twist Angle: This time the sample cell was a $90^{\circ} \mathrm{TN}$ cell, then the retardation can be determined by solving (7c) with known $\chi$. The characteristic angle was found to be also $-85^{\circ}$ by the iterative method. Then from Fig. 5, the retardation was determined to be $1158.5 \mathrm{~nm}$.

\section{Stokes Parameter Method for GTN Cell PARAMETERS MEASUREMENT}

In this section, we are going to show that if a Stokesmeter (or polarimeter) is available, then the cell parameters $(\phi, d \Delta n)$ can be determined much easier. No iterative process is involved in $\chi$ determination and the phase $\Gamma$ can be determined without waveplate insertion and careful alignment as in the modified Senarmont method. Before going into the measurement method details, some background knowledge is required. Below we shall introduce the concept of characteristic circle and ellipse [24].

Suppose we have a measurement setup as shown in Fig. 6. The Stokesmeter can display the Stokes parameters of the output light $S$ instantly. While the GTN cell is rotating, the locus of the point $\left(S_{1}, S_{2}\right)$ on the $S_{1}-S_{2}$ plane always traces a circle. We call

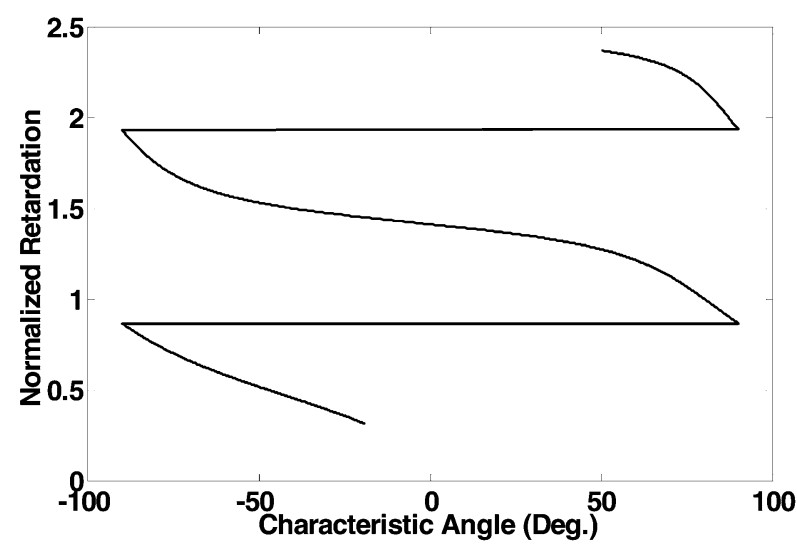

Fig. 5. For known twist angle, the retardation value can be determined from the characteristic angle alone. The plot of (7c) with twist angle $\phi=90^{\circ}$ is shown here.

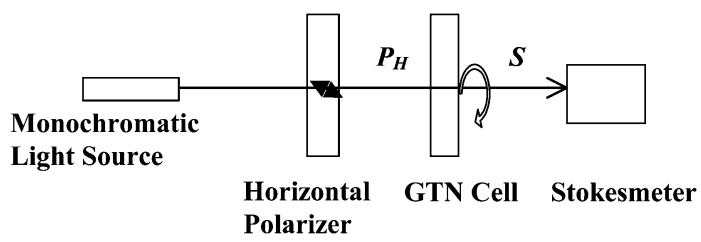

Fig. 6. A schematic of the experimental setup for characteristic circle measurement. For characteristic ellipse measurement, a polarization rotator is inserted between the input polarizer and the sample cell.

this the characteristic circle. A proof of that above statement is as follows.

Proof: Suppose the GTN cell is represented by a waveplate followed by a rotator, then it is obvious that the shape of the $\left(S_{1}, S_{2}\right)$ locus does not depend on the rotator angle. For simplicity, let us assume that the rotator value is zero, then the final Stokes vector is calculated by

$$
S=M_{\text {rot }}(\theta) \bullet M_{W P}(\Gamma) \bullet M_{\text {rot }}(-\theta) \bullet P_{H}
$$

where $M_{\text {rot }}(\theta)$ represents the Mueller matrix [22] of a polarization rotator with angle $\theta, M_{W P}(\Gamma)$ represents the waveplate with phase angle $\Gamma, P_{H}$ is the input horizontal polarized light and $S$ is the final Stokes vector. According to (8), $S$ is

$$
S=\left(\begin{array}{c}
\cos ^{2} 2 \theta+\sin ^{2} 2 \theta \cos \Gamma \\
\sin 2 \theta \cos 2 \theta(1-\cos \Gamma) \\
\sin \Gamma \sin 2 \theta
\end{array}\right) .
$$

Here we have neglected the $S_{\mathrm{o}}$ of the Stokes vector which is assumed to be equal to one for a lossless unitary media. Eliminating $\theta$ from $S_{1}$ and $S_{2}$ gives

$$
\left(S_{1}-\frac{1+\cos \Gamma}{2}\right)^{2}+S_{2}^{2}=\left(\frac{1-\cos \Gamma}{2}\right)^{2}
$$

which clearly shows the equation of a circle on the $S_{1}-S_{2}$ plane. The equation describes circles with azimuth angle at zero and radius and center depends on the phase angle of the equivalent waveplate. The radius of the circle is $(1-\cos \Gamma) / 2$ or simply $\sin ^{2} \Gamma / 2$, which is equal to the left-hand side of $(7 b)$.

For a twisted nematic LC cell, the azimuth of the characteristic circle is no longer zero in general. In fact the azimuthal angle is equal to the characteristic angle $\chi$ of the GTN cell. Therefore by recording the locus of the $\left(S_{1}, S_{2}\right)$ point, $(\phi, d \Delta n)$ 
TABLE I

Measurement Results of Two lCD Samples by the Characteristic Circle and Ellipse, Respectively. Here $\langle R\rangle$ is the Average Radius of the Characteristic Circle and $\langle e\rangle$ IS THE AVERAge ElLIPTICITY OF THE CHARACTERISTIC ElLIPSE. INPUT WAVELENGTH IS $632.8 \mathrm{~nm}$

\begin{tabular}{lcccccc}
\hline & \multicolumn{2}{c}{ Characteristic Circle } & \multicolumn{3}{c}{ Characteristic Ellipse } \\
\hline & $<\boldsymbol{R}>$ & $\chi$ & $(\boldsymbol{\phi}, \boldsymbol{d} \Delta \boldsymbol{n})$ & $<\boldsymbol{e}$ & $\chi$ & $(\boldsymbol{\phi}, \boldsymbol{d} \boldsymbol{\Delta n})$ \\
$\boldsymbol{T N}$ cell & 0.098 & $84.88^{\circ}$ & $\left(89.9^{\circ}, 1157 \mathrm{~nm}\right)$ & 0.826 & $85.4^{\circ}$ & $\left(89.8^{\circ}, 1161 \mathrm{~nm}\right)$ \\
STN cell & 0.18 & $85.77^{\circ}$ & $\left(241.1^{\circ}, 735 \mathrm{~nm}\right)$ & 0.612 & $85.6^{\circ}$ & $\left(241^{\circ}, 732 \mathrm{~nm}\right)$ \\
\hline
\end{tabular}

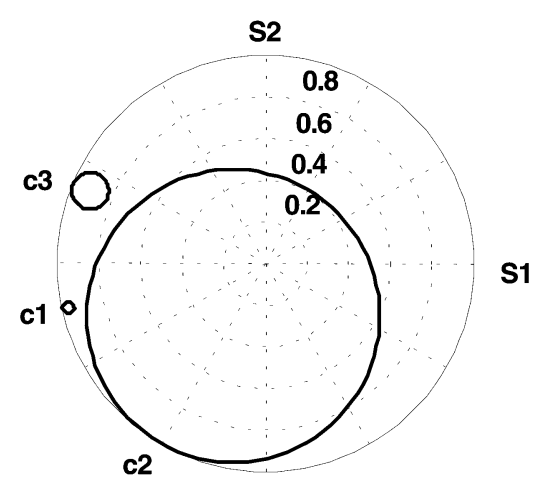

Fig. 7. Representative circles drawn on the $S_{1}-S_{2}$ plane for first minimum $\mathrm{TN}\left(\phi=90^{\circ}, d \Delta n=0.5 \mu \mathrm{m}\right) \mathrm{C} 1$, second minimum $\mathrm{TN}\left(\phi=90^{\circ}, d \Delta n=\right.$ $1.0 \mu \mathrm{m}) \mathrm{C} 2$ and the STN $\left(\phi=240^{\circ}, d \Delta n=0.85 \mu \mathrm{m}\right) \mathrm{C} 3$. Wavelength used is $632.8 \mathrm{~nm}$.

of the GTN cell can be obtained. Fig. 7 shows some typical characteristic circles.

One problem of the above characteristic circle method is that, sometimes it is not convenient or even not possible to rotate the sample under investigation. A solution to the above problem is to rotate the input light polarization instead of the sample itself. Note that under this arrangement, the $\left(S_{1}, S_{2}\right)$ locus is now no longer a circle, but an ellipse in general. This fact is revealed by considering the GTN cell as a waveplate. The resulting equation is

$$
\left(\begin{array}{l}
S_{1} \\
S_{2} \\
S_{3}
\end{array}\right)=\left(\begin{array}{ccc}
1 & 0 & 0 \\
0 & \cos \Gamma & -\sin \Gamma \\
0 & \sin \Gamma & \cos \Gamma
\end{array}\right)\left(\begin{array}{c}
\cos 2 \theta \\
\sin 2 \theta \\
0
\end{array}\right)
$$

where $\theta$ represents the input polarizer direction.

Then we have

$$
\begin{aligned}
& S_{1}=\cos 2 \theta \\
& S_{2}=\cos \Gamma \sin 2 \theta .
\end{aligned}
$$

Eliminate $\theta$ from $S_{1}$ and $S_{2}$ gives

$$
S_{1}^{2}+\left(\frac{S_{2}}{\cos \Gamma}\right)^{2}=1
$$

Equation (13) is the equation of an ellipse in the $S_{1}-S_{2}$ plane with ellipticity $\boldsymbol{e}=\cos \Gamma$. Therefore, by measuring ellipticity of the resulting ellipse, one can obtain the characteristic phase $\Gamma$. The characteristic angle $\chi$ is obtained by recording $\theta$ for a linear output with and without the sample. The angle between the two is the characteristic angle $\chi$. Some typical characteristic ellipses are shown in Fig. 8.

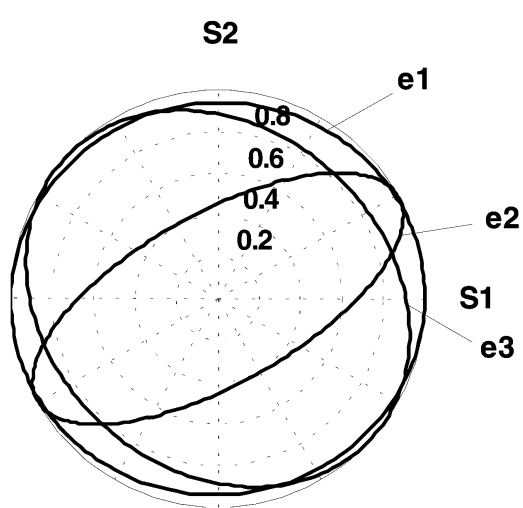

Fig. 8. Representative ellipses drawn on the $S_{1}-S_{2}$ plane for first minimum $\mathrm{TN}\left(\phi=90^{\circ}, d \Delta n=0.5 \mu \mathrm{m}\right) \mathrm{e} 1$, second minimum $\mathrm{TN}\left(\phi=90^{\circ}, d \Delta n=\right.$ $1.0 \mu \mathrm{m}) \mathrm{e} 2$ and the STN $\left(\phi=240^{\circ}, d \Delta n=0.82 \mu \mathrm{m}\right) \mathrm{e} 3$. Wavelength used is $632.8 \mathrm{~nm}$.

TABLE II

SUMMARY OF THE RELATIONS BETWEEN VARIOUS CHARACTERISTIC PARAMETERS AND THE GTN CELl PARAMETERS

\begin{tabular}{l|c}
\hline \multicolumn{1}{c|}{ Characteristic Parameter } & Relation to GTN Cell Parameters \\
\hline Characteristic Direction $\alpha$ & $\tan 2 \alpha=(\phi / \beta) \tan \beta$ \\
\hline Characteristic Angle $\chi$ & $\begin{array}{l}\tan \chi=(\beta \sin \phi \cos \beta-\phi \cos \phi \sin \beta) / \\
(\beta \cos \phi \cos \beta+\phi \sin \phi \sin \beta)\end{array}$ \\
\hline Characteristic Phase $\Gamma$ & $\sin ^{2}(\Gamma / 2)=\left(\delta^{2} / \beta^{2}\right) \sin ^{2} \beta$ \\
\hline Characteristic Circle Radius $R$ & $R=\left(\delta^{2} / \beta^{2}\right) \sin ^{2} \beta$ \\
\hline Characteristic Ellipse Ellipticity $\boldsymbol{e}$ & $e=1-2\left(\delta^{2} / \beta^{2}\right) \sin ^{2} \beta$ \\
\hline
\end{tabular}

Example 3: TN and STN Cells: The STN and TN cell measured in Examples 1 and 2 were measured again by the Stokes parameter method. The Stokesmeter used was a compact rotating QWP [22] type polarimeter from Thorlab Inc. For the characteristic ellipse measurement, an ECB (electrically controlled birefringence) LC cell plus a QWP were used to act as the input polarization rotator. The results are listed in Table I. They are very close to that obtained by previous methods.

\section{CONCLUSIONS}

In this paper, we have introduced the concept of characteristic parameters for LC cells. In Section II, we obtained the relations between the characteristic parameters of the optically equivalent model and the LC cell parameters of a twisted nematic 
cell. Then, in Section III, methods for the determination of the characteristic parameters are described. Once the characteristic parameters are known, the LC cell twist angle and retardation can be solved. Illustrative examples are given in Section IV. A method for the effective removal of solution ambiguity is also introduced. At last, in Section V, two new characteristic parameters, the characteristic circle and the characteristic ellipse are introduced. They are used in the Stokes parameter method when a Stokesmeter (or polarimeter) is available. This method has the advantages of simple operation.

A summary of the various characteristic parameters used and their relation to the LC cell parameters are listed in Table II. The accuracy of the methods described in Section III is expected to be around $1^{\circ}$ for the twist angle and $2 \mathrm{~nm}$ for the retardation value in typical TN/STN cells. This corresponds to a $1^{\circ}$ uncertainty in the characteristic phase angle $\Gamma$ determination and/or the characteristic rotator angle $\chi$. The accuracy of the characteristic circle and ellipse methods are similar (Fig. 4).

Finally, it is worth mentioning that for GTN cells with internal reflector such as the silicon microdisplays, the equivalent model shows that the resultant matrix is independent of the rotator angle $\chi$, therefore we have only (7a) and (7b). As has been mentioned, if the input director orientation is unknown, we cannot solve for both twist angle and the retardation. In common practice, the twist angle is known, and then the input director orientation is also known, then the retardation can be solved easily from (7a) [9].

\section{REFERENCES}

[1] S. Huard, Polarization of Light. New York: Wiley, 1996

[2] H. Hurwitz and R. C. Jones, "A new calculus for the treatment of optical systems-II: Proof of three general equivalent theorems," J. Opt. Soc. Amer., vol. 31, pp. 493-499, Jul. 1941.

[3] S. T. Tang and H. S. Kwok, "A new method to measure twist angle and cell gap of LC cells," in SID '98 Dig., 1998, pp. 552-555.

[4] M. Bosma, Method of Determination of Retardation and Twist Angle of Twisted Birefringent Layers. Deventer, The Netherlands: Akzo Nobel, 1997.

[5] T. Inoue, "Method of measuring thickness of liquid crystal cells," U.S. Patent 5239365, Aug. 24, 1993.

[6] A. Lien and H. Takano, "Cell gap measurement of filled twisted nematic liquid crystal displays by a phase compensation method," J. Appl. Phys., vol. 69, pp. 1304-1309, 1991.

[7] A. Lien, "Simultaneous measurement of twist angle and cell gap of a twisted nematic cell by an optical method," in IDRC '91, 1991, pp. 192-194.

[8] X. Shao, T. Yu, Z. Wang, J. Yuan, J. Guo, and X. Huang, "A new method to measure thickness of twist nematic liquid crystal cells," in EuroDisplay '96, 1996, pp. 309-312.

[9] S. T. Tang and H. S. Kwok, "Reflective method of LCD measurement," in $I D W^{\prime} 00,2000$, pp. 109-111.
[10] - "Transmissive liquid crystal cell parameters measurement by spectroscopic ellipsometry," J. Appl. Phys., vol. 89, pp. 80-85, 2001.

[11] L. S. Srinath and A. V. S. S. S. R. Sarma, "Determination of the optically equivalent model in three-dimensional photoelasticity," Experimental Mechanics, vol. 14, pp. 118-122, 1974.

[12] L. S. Srinath, K. Ramesh, and V. Ramamurti, "Determination of characteristic parameters in three-dimensional photoelasticity," Optical Engineering, vol. 27, pp. 225-230, 1988.

[13] H. Aben, Integrated Photoelasticity. New York: McGraw-Hill, 1979.

[14] - "Characteristic directions in optics of twisted birefringence media," J. Opt. Soc. Amer. A, vol. 3, pp. 1414-142, 1986.

[15] - " "Optical phenomena in photoelastic models by the rotation of principal axes," Experimental Mechanics, vol. 6, pp. 13-22, 1966.

[16] S. Chandrasekhar, Liquid Crystals, 2nd ed. New York: Cambridge Univ. Press, 1992.

[17] E. P. Raynes and R. J. A. Tough, "The guiding of plane polarized light by twisted liquid crystal layers," Mol. Cryst. Liq. Cryst. Lett., vol. 2, pp. 139-145, 1985.

[18] A. Yariv and P. Yeh, Optical Waves in Crystals-Propagation and Control of Laser Radiation. New York: Wiley, 1984.

[19] P. Yeh and C. Gu, Optics of Liquid Crystal Displays. New York: Wiley, 1999.

[20] L. S. Srinath and S. Y. Keshavan, "A fast iterative procedure to determine photoelastic characteristic parameters," Mech. Res. Commun., vol. 5, pp. 159-165, 1978.

[21] I. C. Khoo and S. T. Wu, Optics and Nonlinear Optics of Liquid Crystals: World Scientific, 1993.

[22] E. Collett, Polarized Light-Fundamentals and Applications. Reading, MA: Marcel Dekker, 1992.

[23] S. T. Tang, "Polarization optics of liquid crystal and its applications," Ph.D. disertation, Hong Kong Univ. of Science and Technology, 2001.

[24] S. T. Tang and H. S. Kwok, "3 33 matrix for unitary optical systems," J. Opt. Soc. Amer. A, vol. 18, pp. 2138-2145, 2001

S. T. Tang received the B.S. degree in physics from the National Taiwan University in 1988 and the M.S. and Ph.D. degrees in electrical and electronic engineering from the Hong Kong University of Science and Technology in 1995 and 2001, respectively.

He joined Philips Mobile Display System in 2001. In 2002 he joined Bona Fide Instruments Company Ltd., as the Chief Technical Officer, where he has been working on the development of display test and measurement systems.

Dr. Tang is a member of the Society for Information Display (SID) and SPIE.

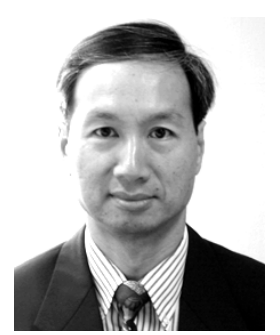

Hoi-Sing Kwok (S'73-M'78-SM'84-F'03) received the Ph.D. degree in applied physics from Harvard University, Cambridge, MA, in 1978.

$\mathrm{He}$ joined the State University of New York at Buffalo in 1980 and became Full Professor in 1985. He joined Hong Kong University of Science and Technology (HKUST) in 1992 and is currently Chair Professor and Director of the Center for Display Research. He has over 500 refereed publications and hold over 20 patents in laser optics and LCD technologies.

Dr. Kwok is a Fellow of the Optical Society of America, and a Fellow of Society for Information Display (SID). He is also an Elected Member of the Asia Pacific Academy of Materials. 